\title{
Niveles de Actividad Física y sus Determinantes en Mujeres Adultas de Barranquilla
}

\section{Determinant Factors in Physical Activity Levels in Adult Women in Barranquilla}

Recibido: 5 de Oct. 2013/Enviado para modificación: 10 de Nov. 2013/Aceptado: 14 de En. 2014

\author{
Yisel Pinillos Patiño ${ }^{1}$ \\ Universidad Simón Bolívar \\ Yaneth Herazo Beltrán 2 \\ Universidad Simón Bolívar \\ Jose Armando Vidarte Claros ${ }^{3}$ \\ Universidad Autónoma de Manizales \\ Estela Crissien Quiroz ${ }^{4}$ \\ Universidad Simón Bolívar \\ Damaris Suarez Palacio 5 \\ Universidad Simón Bolívar
}

\section{RESUMEN}

Introducción: Un factor personal relacionado con la actividad física es el género. Es relevante comprender al respecto los factores que influyen en los niveles de actividad física en un grupo de mujeres. Objetivo: Identificar los factores que determinan los niveles de actividad física en mujeres adultas de Barranquilla. Materiales y Métodos: Se realizó un estudio descriptivo transversal en 1067 mujeres entre 18 y 65 años de edad, mediante un muestreo probabilístico y multietápico. La actividad física se evaluó a través del Cuestionario Internacional de Actividad Física (IPAQ), formato corto. Con el paquete estadístico SPSS, se definieron las frecuencias de las variables estudiadas y su relación a través de la prueba chi2 y el OR como indicador de riesgo. Resultados: el 61,7\% de las mujeres declaró ser activa físicamente. Se encontró un mayor riesgo de no realización de actividad física en el grupo de mujeres casadas o en unión libre [OR 1,4 IC 95\% (1,1-1,9)], en las que presentan un nivel de escolaridad ninguno o primaria incompleta, primaria completa o secundaria completa o incompleta [OR 1,6 IC 95\% (1,2-2,1)], las que se ubican en las etapas de precontemplación, contemplación, preparación y recaída [OR 2,6 IC 95\% (1,9-3,5)], y en aquellas que perciben su salud como mala y regular [OR 1,5 IC 95\% (1,1-2)]. Conclusiones: La actividad física en las mujeres de Barranquilla está relacionada con el rango de edad, el estado civil, el nivel educativo y la localidad donde residen. Asimismo, la falta de voluntad para realizar actividad física, las etapas de cambio y la autopercepción de salud están relacionadas con el hecho de ser activas e inactivas físicamente.

Palabras Clave: actividad motora, salud de la mujer, factores socioeconómicos (Fuente: DeCS).

\section{ABSTRACT}

Introduction: The genre is a personal factor related to physical activity. It To understand the factors that influence the levels of physical activity in a group of women would be relevant. Objective: To identify factors that determine the levels of physical activity in adult women from Barranquilla. Materials and Methods: 1067 women between 18 and 65 years of age were sampled in a transversal and descriptive. Physical activity was assessed through the International Questionnaire on Physical activity (IPAQ), short form. By means of the statistical bundle SPSS there were defined the frequencies of the studied variables and its relation using the test chi2 and the OR as risk indicators. Results: $61,7 \%$ is physical active. A risk of not doing physical activity in group of married women or in a living together type relationship was found [OR 1,4 IC 95\% (1,1-1,9)], in those that are in school , or contemplating enrolling in or returning to some sort of educational institution [OR 2,6 IC 95\% (1,9-3,5)], and in those that have a certain level of schooling, none or incomplete primary or completed, or completed secondary or incompleted [OR 1,6 IC 95\% (1,2-2,1)], [OR 2,6 IC 95\% (1,9-3,5)], and in those that perceive his health as bad and regular [OR 1,5 IC 95\% (1,1-2). Conclusions: Physical activity in women of Barranquilla is related to the age status, marital status, educational level and locality. Likewise, the barrier the lack of will power to cattery out a physical activity as well as a stage of change and their perception of good health are factors in determining whether to be active or inactive.

Keywords: motor activity, women health, socioeconomic factors (Source: MeSH, NLM).

Para citar este artículo: Pinillos PY, Herazo BY, Vidarte CJ, Crissien QE, Suarez PD. Niveles de actividad física y sus determinantes en mujeres adultas de Barranquilla. Cienc. innov. salud. 2014; 2 (1): 11-17.

\footnotetext{
Fisioterapeuta. MSc Salud Pública. Universidad Simón Bolívar. Correo electrónico: ypinillos@ unisimonbolivar.edu.co Fisioterapeuta. MSc Salud Pública. Universidad Simón Bolívar. Correo electrónico: aherazo4@unisimonbolivar.edu.co Licenciado en Educación Física. Doctor en Ciencias de la Actividad Física y el Deporte. Universidad Autónoma de Manizales. Fisioterapeuta. Magister en Educación. Universidad Simón Bolívar. Correo electrónico: ecrissien@unisimonbolivar.edu.co Fisioterapeuta. MSc Salud Pública. Universidad Simón Bolívar. Correo electrónico: dsuarez3@unisimonbolivar.edu.co
} 


\section{Introducción}

Las enfermedades cardiovasculares se han reconocido mundialmente como la principal causa de morbilidad y mortalidad. Por otra parte, los estilos de vida saludables generalmente se relacionan con la vulnerabilidad frente a los factores de riesgo para la salud, que enfrenta la persona en el contexto en el cual se desarrolla (1).

En este sentido, la OMS identifica la inactividad física como el cuarto factor de riesgo de las muertes a nivel mundial $(6 \%)$. Y lo grave es que por lo menos el $60 \%$ de la población no realiza la actividad física necesaria para contar con una buena salud, estimándose que ello es la causa de un 21\%-25\% de los cánceres de mama y colon, el $27 \%$ de los casos de diabetes y de, aproximadamente, el $30 \%$ de las cardiopatías isquémicas (2). De manera concreta, otros indicadores informan que el $27 \%$ de los adultos en Estados Unidos no realizan ninguna actividad física; y que en Europa los países mediterráneos son los más sedentarios, estilo de vida este que se relaciona con características tales como la obesidad, bajo nivel educativo y tabaquismo (3). En el caso de Colombia, la identificación de los niveles de actividad física como una necesidad de salud pública se ha obtenido a partir de estudios regionales, los cuales también se asocian a los factores de riesgo presentes en la población mundial (4).

Con base en lo anterior, se puede decir que los niveles de inactividad física son elevados en prácticamente todos los países desarrollados y en desarrollo, lo que constituye un problema de salud pública, sobre todo en poblaciones en riesgo como jóvenes, mujeres y adultos mayores. Este problema se acrecienta en las ciudades de rápido crecimiento, debido a factores como la urbanización, la superpoblación, el aumento de la pobreza, el aumento de la criminalidad e inseguridad, la alta densidad del tráfico automotriz, la mala calidad del ambiente y la inexistencia de una infraestructura adecuada para la realización de la actividad física.

De igual manera, la práctica de la actividad física está determinada por patrones individuales de conducta, tales como sexo, edad, niveles de destrezas, habilidades y discapacidades, comportamientos, actitudes y motivaciones (5).
A partir de esto último, cabe decir que el estudio de las diferencias existentes entre personas de género masculino y femenino con respecto a la práctica de actividad física, está bastante documentado y permite comprender el pensamiento y comportamiento de la población. Poco a poco la actividad física se instaura en la vida de las mujeres; sin embargo, los estudios sugieren que la cantidad de actividad física practicada por el grupo femenino continúa siendo inferior comparado con el de los hombres, lo cual podría estar asociado a normas sociales y patrones culturales (6-9).

Entendida la actividad física como "Cualquier movimiento intencional, realizado con los músculos esqueléticos, que resulta en un gasto de energía y en una experiencia personal y nos permite interactuar con los seres y el ambiente que nos rodea" (10), queda claro que su definición comprende la dimensión biológica, personal y sociocultural, y no tan solo el aspecto del movimiento corporal intencionado que genera gasto energético.

En este orden de ideas, la actividad física como factor determinante de la salud tiene repercusiones importantes, las cuales se evidencian en los resultados de investigaciones científicas, que reafirman los beneficios que esta produce en la salud física y mental de las personas, al generar efectos fisiológicos positivos sobre los sistemas corporales y el funcionamiento de los sistemas metabólicos. Todo ello produce un gran impacto en la reducción de la mortalidad por causa de los factores de riesgo, pues previene el riesgo de padecer enfermedades crónicas coronarias, vasculares y osteoarticulares, así como enfermedades mentales y algunos tipos de cáncer. Conjuntamente, la actividad física mejora los síntomas de las deficiencias sistémicas presentes en el individuo, mantiene su funcionalidad y limita al máximo la dependencia (3).

Por esta razón, como primer elemento para el desarrollo de acciones de promoción de hábitos de vida saludables, se requiere establecer líneas de base con el fin de caracterizar los comportamientos de salud en la población. Y, con especial cuidado deben tomarse en cuenta los factores que influyen en el comportamiento de las mujeres frente a la práctica de la actividad física. Precisamente por esto último, en esta investigación se buscó determinar el nivel de actividad física de un grupo de mujeres adultas de la ciudad de Barranquilla. 


\section{Materiales y Métodos}

El presente trabajo es en sí resultado de un estudio descriptivo transversal que se realizó en las cinco localidades de Barranquilla: Río-Mar, Norte CentroHistórico, Suroccidente, Suroriente y Metropolitana. Este estudio hace parte de la investigación de base poblacional sobre los factores asociados a los niveles de actividad física en adultos de la ciudad.

En específico, mediante un muestreo probabilístico y multietápico, se seleccionó una muestra de 1067 mujeres entre 18 y 65 años de edad. Esta muestra fue escogida teniendo en cuenta que el $51,9 \%$ de la población total de Barranquilla está constituida por mujeres, de acuerdo con las proyecciones del DANE para el año 2011, que comprendían un total de 620.855 mujeres. Al ser un muestreo multietápico, el acercamiento a la población de estudio se realizó a partir de la localidad, el barrio, la calle y la vivienda donde habitara una mujer que se encontrara entre las edades referenciadas.

Se aplicó un instrumento que indagó sobre los siguientes elementos: género, edad, estrato socioeconómico, nivel educativo y estado civil; la intención de práctica, considerando las seis etapas de cambio: precontemplación, contemplación, preparación, acción, mantenimiento y recaída; y autopercepción del estado salud, que propone cuatro respuestas: muy buena, buena, regular y mala. La precontemplación se refiere a personas que no realizan actividad física ni tienen intención de práctica en un lapso de seis meses; la contemplación incluye a personas que no realizan actividad física, pero tienen intención de hacerla en los próximos seis meses; la preparación considera a personas que no realizan actividad física, pero que tienen intención de hacerla en los próximos 30 días; la acción, a personas que realizan actividad física regular desde hace menos de seis meses; el mantenimiento incluye a personas que manifiestan realizar actividad física regular desde hace más de seis meses; por último, la recaída involucra a personas que realizaban actividad física de forma regular, pero la abandonaron recientemente.

Para definir los niveles de actividad física de las mujeres, se usó el Cuestionario Internacional de Actividad Física (IPAQ), formato corto. En el nivel bajo, se consideraron los casos en que no se reportaba actividad o que no podían incluirse en niveles

Cienc. innov. salud. Junio 2014; 2 (1):11 - 17. Universidad Simón Bolívar (Col). ISSN: 2344-8636 http://portal.unisimonbolivar.edu.co:82/rdigital/innovacionsalud moderado o alto. En el nivel regular, se clasificó a personas que reportaron cualquiera de las siguientes situaciones: 3 o + días de actividad intensa de al menos 20 minutos por día; o 5 o más días de actividad moderada y/o caminata de al menos 30 minutos; así como 5 o más días de cualquier combinación de caminata, moderada o intensa, que llegara a 600 METS-minutos por semana. Y el nivel alto o intenso contempló a cualquiera de los 2 criterios siguientes: 3 días que acumularan actividad física intensa de 1500 METS-minuto por semana; 7 o más días de cualquier combinación (caminata, moderada, intensa) que acumulara 3000 METS-minutos por semana.

Los sujetos de estudio diligenciaron el consentimiento informado, en el cual se les explicó los objetivos del estudio y en qué consistía su participación. Y para el procesamiento de los datos y análisis de la información, se utilizó el paquete estadístico SPSS versión 18.0 (Licencia $\mathrm{N}^{\circ}$ 10138194). Mediante este último, se realizó un análisis bivariado para estimar las relaciones entre los niveles de actividad física y las características sociodemográficas, autopercepción de salud y etapas de cambio, usando la prueba Chi2 para determinar la significancia estadística. Igualmente, se realizó un análisis de regresión logística, usando como indicador de riesgo el odds ratio.

\section{Resultados}

En la Tabla 1, se puede apreciar la frecuencia de las variables sociodemográficas. En este sentido, se resalta que el $42,8 \%$ de las mujeres encuestadas tienen entre 18 y 29 años; el 78,6\% pertenecen a los estratos 1,2 y 3 ; y la mayoría reside en el sur de la ciudad. La media de edad fue de 35,2 $\pm 13,7$ años. La prevalencia de actividad física encontrada en el estudio fue de $61,7 \%$; aun cuando se observa que sólo el 5,7\% de las mujeres sobrepasan la práctica regular de actividad física.

Tabla 1. Características generales de las participantes

\begin{tabular}{clcc}
\hline Variable & $\begin{array}{c}\text { Niveles de la } \\
\text { Variable }\end{array}$ & Frec. Obs. & Rep. \% \\
\hline \multirow{3}{*}{ Rango de edad } & $18-29$ años & 457 & $42,80 \%$ \\
\cline { 2 - 4 } & $30-49$ años & 412 & $38,60 \%$ \\
\cline { 2 - 4 } & 50-65 años & 198 & $18,60 \%$ \\
\hline
\end{tabular}


14 Yisel Pinillos Patiño, Yaneth Herazo Beltrán, Jose Vidarte Claros, Estela Crissien Quiroz \& Damaris Suarez Palacio

\begin{tabular}{|c|c|c|c|c|c|c|c|c|}
\hline Variable & $\begin{array}{c}\text { Niveles de la } \\
\text { Variable }\end{array}$ & Frec. Obs. & Rep. \% & $\begin{array}{l}\text { Tabla 2. Niv } \\
\text { socio-demog }\end{array}$ & $\begin{array}{l}\text { les de act } \\
\text { áficas }\end{array}$ & vidad físi & a según $v$ & riables \\
\hline \multirow{4}{*}{ Estado civil } & Soltera & 465 & $\begin{array}{l}43,60 \% \\
19,70 \%\end{array}$ & \multirow{2}{*}{$\begin{array}{c}\text { Variables } \\
\text { socio- } \\
\text { demográficas }\end{array}$} & \multirow{2}{*}{$\begin{array}{c}\begin{array}{c}\text { Niveles } \\
\text { de las } \\
\text { Variables }\end{array} \\
\end{array}$} & \multicolumn{2}{|c|}{$\begin{array}{c}\text { Niveles de Actividad } \\
\text { Física }\end{array}$} & \multirow{2}{*}{ p-Valor } \\
\hline & $\begin{array}{l}\text { Casada } \\
\text { Unión libre }\end{array}$ & $\frac{210}{289}$ & $\begin{array}{l}19,10 \% \\
27.10 \%\end{array}$ & & & Inactivos & Activos & \\
\hline & $\begin{array}{l}\text { Separada/ } \\
\text { divorciada }\end{array}$ & 68 & $6,40 \%$ & \multirow{3}{*}{ Rango de edad } & \multirow{3}{*}{$\begin{array}{l}18-29 \\
\text { años } \\
30-49 \\
\text { años } \\
50-65 \\
\text { años } \\
\end{array}$} & $163(35,7)$ & $294(64,3)$ & \multirow{3}{*}{0,000} \\
\hline & Viuda & 35 & $3,30 \%$ & & & $170(41,3)$ & $\begin{array}{l}242 \\
(58.72)\end{array}$ & \\
\hline \multirow{5}{*}{ Nivel Educativo } & $\begin{array}{l}\text { Ninguno o } \\
\text { primaria }\end{array}$ & 82 & $7,70 \%$ & & & $103(52)$ & $\begin{array}{l}(58,72) \\
95(48)\end{array}$ & \\
\hline & $\begin{array}{l}\text { 1ncompleta } \\
\text { Primaria } \\
\text { completa }\end{array}$ & 144 & $13,50 \%$ & \multirow[b]{2}{*}{ Estado civil } & \multirow{2}{*}{$\begin{array}{l}\text { Casada } \\
\text { /Unión } \\
\text { libre } \\
\text { Soltera/Se } \\
\text { parada/Di } \\
\text { vorciada/ } \\
\text { Viuda } \\
\end{array}$} & $239(44,4)$ & $299(55,6)$ & \multirow[b]{2}{*}{0,01} \\
\hline & $\begin{array}{l}\text { Secundaria } \\
\text { completa o } \\
\text { incompleta }\end{array}$ & 512 & $48,00 \%$ & & & $197(37,2)$ & $332(62,8)$ & \\
\hline & $\begin{array}{l}\text { Estudios } \\
\text { superiores }\end{array}$ & 304 & $28,50 \%$ & \multirow{9}{*}{$\begin{array}{l}\text { Nivel } \\
\text { educativo }\end{array}$} & \multirow{9}{*}{$\begin{array}{l}\text { Ninguno o } \\
\text { primaria } \\
\text { incompleta/ } \\
\text { Primaria } \\
\text { completa/ } \\
\text { Secundaria } \\
\text { completa o } \\
\text { incompleta } \\
\text { Secundaria } \\
\text { completa o } \\
\text { incompleta/ } \\
\text { Postgrado } \\
\end{array}$} & & & \multirow{9}{*}{0,001} \\
\hline & Postgrados & 25 & $2,30 \%$ & & & & & \\
\hline \multirow{6}{*}{$\begin{array}{l}\text { Estrato } \\
\text { Socioeconómico }\end{array}$} & Estrato 1 & 468 & $43,90 \%$ & & & $328(44,4)$ & $410(55,6)$ & \\
\hline & Estrato 2 & 179 & $16,80 \%$ & & & & & \\
\hline & Estrato 3 & 191 & $17,90 \%$ & & & & & \\
\hline & Estrato 4 & 160 & $15,00 \%$ & & & & & \\
\hline & Estrato 5 & 40 & $3,70 \%$ & & & & & \\
\hline & Estrato 6 & 29 & $2,70 \%$ & & & $108(32,8)$ & $221(67,2)$ & \\
\hline \multirow{5}{*}{ Localidad } & Riomar & 66 & $6,20 \%$ & & & & & \\
\hline & $\begin{array}{l}\text { Norte-centro } \\
\text { histórico }\end{array}$ & 211 & $19,80 \%$ & $\begin{array}{l}\text { Estrato Socio- } \\
\text { económico }\end{array}$ & $\begin{array}{l}1 / 2 / 3 \\
4 / 5 / 6 \\
\end{array}$ & $\begin{array}{l}334(39,9) \\
102(44,5)\end{array}$ & $\begin{array}{l}504(60,1) \\
127(55,5) \\
\end{array}$ & 0,20 \\
\hline & Suroccidente & 342 & $32,10 \%$ & \multirow{6}{*}{ Localidad } & \multirow{6}{*}{$\begin{array}{l}\text { Río-mar } \\
\text { Norte } \\
\text { centro- } \\
\text { histórico } \\
\text { Sur- } \\
\text { occidente } \\
\text { Suroriente } \\
\text { Metropolita } \\
\text { na }\end{array}$} & $24(36,4)$ & $42(63,6)$ & \multirow{6}{*}{0,0000} \\
\hline & Suroriente & 242 & $22,70 \%$ & & & $63(29,9)$ & $148(70,1)$ & \\
\hline & Metropolitana & 206 & $19,30 \%$ & & & & & \\
\hline \multirow{3}{*}{$\begin{array}{l}\text { Niveles de } \\
\text { actividad física }\end{array}$} & Alto & 61 & $5,70 \%$ & & & $190(55,6)$ & $152(44,4)$ & \\
\hline & Regular & 597 & $56 \%$ & & & $87(36)$ & $155(64)$ & \\
\hline & Bajo & 409 & $38,30 \%$ & & & $72(35)$ & $134(65)$ & \\
\hline
\end{tabular}

Por su parte, la Tabla 2 presenta los resultados de las pruebas chi2 al relacionar los niveles de actividad física y las variables socio-demográficas. Se encontraron diferencias significativas entre rango de edad, estado civil, nivel educativo y la localidad de los sujetos, y el hecho de ser activo o inactivo físicamente. También se observa que hay un mayor porcentaje $(46,5 \%)$ de mujeres inactivas mayores de 50 años, en contraste con las menores de 29 años, con un $32,6 \%$.

En la Tabla 3, se observa una diferencia significativa entre las mujeres activas e inactivas en cuanto a las etapas de cambio y la autopercepción de su salud. El porcentaje de mujeres activas en las etapas Acción y Mantenimiento es mayor que en las de Precontemplación, Contemplación, Preparación y Recaída $(\mathrm{p}<0,05)$. Igualmente, hay más personas inactivas $(48,7 \%)$ que perciben su salud como mala y regular $(\mathrm{p}<0,05)$.

Cienc. innov. salud. Junio 2014; 2 (1):11 - 17. Universidad Simón Bolívar (Col). ISSN: 2344-8636 http://portal.unisimonbolivar.edu.co:82/rdigital/innovacionsalud 
Tabla 3. Relación entre los niveles de actividad física y etapas de cambio y autopercepción de salud

\begin{tabular}{|c|c|c|c|c|}
\hline \multirow{2}{*}{ Variable } & \multirow{2}{*}{ Niveles Variables } & \multicolumn{2}{|c|}{$\begin{array}{c}\text { Niveles de Actividad } \\
\text { Física } \\
\end{array}$} & \multirow[t]{2}{*}{ p-Valor } \\
\hline & & Inactivos & Activos & \\
\hline \multirow[t]{2}{*}{$\begin{array}{l}\text { Etapas de } \\
\text { Cambio }\end{array}$} & $\begin{array}{l}\text { Precontemplación/ } \\
\text { Contemplación/ } \\
\text { Preparación/ } \\
\text { Recaída }\end{array}$ & $\begin{array}{c}363 \\
(46,5)\end{array}$ & $\begin{array}{c}430 \\
(53,5)\end{array}$ & \multirow[t]{2}{*}{0,00} \\
\hline & $\begin{array}{l}\text { Acción/ } \\
\text { Mantenimiento }\end{array}$ & $\begin{array}{c}73 \\
(25,4)\end{array}$ & $\begin{array}{c}214 \\
(74,6)\end{array}$ & \\
\hline \multirow{2}{*}{$\begin{array}{l}\text { Auto- } \\
\text { percepción } \\
\text { de salud }\end{array}$} & $\begin{array}{l}\text { Mala/ } \\
\text { Regular }\end{array}$ & $\begin{array}{c}134 \\
(50,2)\end{array}$ & $\begin{array}{c}133 \\
(49,8)\end{array}$ & \multirow{2}{*}{0,00} \\
\hline & $\begin{array}{l}\text { Buena/ } \\
\text { Muy buena }\end{array}$ & $\begin{array}{c}302 \\
(37,8)\end{array}$ & $\begin{array}{c}498 \\
(62,3)\end{array}$ & \\
\hline
\end{tabular}

Al realizar la regresión logística, se encontró un mayor riesgo de no realización de actividad física en el grupo de mujeres casadas o en unión libre [OR 1,4 IC 95\% (1,1-1,9)]; en las que presentan ningún nivel de escolaridad o primaria incompleta, primaria completa o secundaria completa o incompleta [OR 1,6 IC 95\% $(1,2-2,1)]$; las que se ubican en las etapas de precontemplación, contemplación, preparación y recaída [OR 2,6 IC 95\% (1,9-3,5)]; y en las que perciben su salud como mala y regular [OR 1,5 IC $95 \%(1,1-2)]$.

Tabla 4. Factores asociados a los niveles de actividad física en las mujeres estudiadas

\begin{tabular}{lccc}
\hline Factores asociados & OR & IC 95\% & p-Valor \\
\hline Estrato & 0,53 & $0,36-0,76$ & 0,001 \\
socioeconómico & 1,4 & $1,1-1,9$ & 0,007 \\
Estado civil & 1,6 & $1,2-2,1$ & 0,004 \\
Nivel educativo & 1,5 & $1,1-2$ & 0,006 \\
Percepción de salud & 2,6 & $1,9-3,5$ & 0,000 \\
Etapas de cambio &
\end{tabular}

\section{Discusión}

Al ser las mujeres menos participativas en actividades físicas que los hombres, merece la atención determinar los factores que influyen en este comportamiento. La práctica de actividad física menos frecuente entre las mujeres podría deberse a determinadas normas sociales y culturales, que condicionan a los hombres como individuos más activos físicamente.

Estudios sobre la prevalencia de actividad física y factores relacionados en población entre 18 y 60 años muestran similitudes en cuanto al género. Así, comparadas con los hombres, las mujeres realizan en menor proporción actividad física y en cuanto a los aspectos asociados a la actividad física se observa, de igual forma, que variables como IMC, edad, nivel de escolaridad, estrato y frecuencia de práctica son factores que establecen relaciones de predicción con la inactividad (9-11). Por otra parte, en un estudio realizado solo en mujeres (12), de las 224 mujeres contempladas, el $11 \%$ realizaba actividad física intensa; $10 \%$, moderada; y $30 \%$, insuficiente.

Cabe destacar que, según un estudio realizado en Pereira (Colombia), las mujeres practican más ejercicio físico que los hombres. Pero esto podría deberse a que en esta ciudad existen programas masivos de actividad física creados por políticas gubernamentales (9), a lo que se agrega el programa Risaralda Activa $(13,14)$, que sigue lineamientos para alcanzar tres metas primordiales en las localidades: sensibilización, fase activa y fase de anclaje.

Ahora bien, aunque la prevalencia de la actividad física del presente estudio fue de $45,4 \%$, es importante insistir en la creación de políticas públicas en torno a la práctica de la actividad física y el deporte; ya que otro estudio realizado por la Universidad del Rosario, denominado Muévase contra el Sedentarismo (15), muestra que la mayor parte de la población colombiana no realiza actividad física durante la semana para obtener beneficios en la salud y en la calidad de vida. De igual forma, la prevalencia del sedentarismo en el país resulta alarmante, ya que el $35 \%$ de la población encuestada realiza actividad física una sola vez a la semana, $52,7 \%$ no realiza ninguna actividad, y solo $21,2 \%$ la realiza en forma regular por lo menos tres veces a la semana.

Según Grimaldo, la práctica de actividades deportivas por parte de las mujeres solo se realiza algunas veces, y las posibles causas de ello se deben a mitos como: la masculinización que produce el realizar actividades deportivo-atléticas, la falta de interés por parte de las mujeres y el posible peligro que representa para ellas (16).

Asimismo, en un estudio realizado en Colombia por Robledo Martínez (17) y concomitante a los resultados anteriormente nombrados, las mujeres expresaron que se veían a sí mismas como sedentarias, y los hombres también las percibieron como menos activas que ellos. En un estudio similar sobre la prevalencia de la actividad física en una población de Pamplona (18), se 
pone de manifiesto que el nivel educativo es determinante para predecir el ejercicio, situación que resulta similar a lo encontrado en el presente estudio, ya que estas variables presentaron diferencias estadísticamente significativas (0,001). En ese mismo estudio, se encontró que la edad y el estado civil son los factores que mejor predicen el estilo de vida sedentario en los hombres; y en el caso de las mujeres, el nivel de estudio pareciera ser la mejor variable predictora en relación con un comportamiento inactivo, resultado que al compararse con lo obtenido en el actual estudio muestra diferencias estadísticamente significativas.

Los trabajos que han estudiado los predictores de la actividad física estructuran el análisis según factores personales, sociales y ambientales (19). Desde el punto de vista de los factores personales, se ha encontrado al respecto que la percepción de la competencia física o deportiva se asocia positivamente con la actividad física practicada por las personas, especialmente los adolescentes (21).

En el presente estudio, la barrera falta de voluntad se relacionó con el hecho de ser activo o inactivo; resultados que difieren de los reportados por otros autores, según los cuales el principal motivo de abandono de la práctica de actividad física es la falta de tiempo (7).

En cuanto a la actividad física, estados de cambio y autopercepción, hay estudios que muestran como los beneficios y barreras percibidos por los estudiantes al realizar la actividad física recomendada, indican diferencias según el sexo. En concreto, las mujeres necesitarían un mayor estímulo, lo que plantea un desafío a los encargados de las políticas y programas existentes. La falta de tiempo fue el factor más destacado, y ello evidencia que no es suficiente realizar acciones de promoción. Es preciso analizar los factores ambientales y determinar si las universidades, el entorno que las rodea y las ciudades están ofreciendo la oportunidad de realizar más actividad física, no sólo a los universitarios, sino a toda la población (22).

En lo relacionado con el estado de salud, algunos estudios han reportado la autopercepción del estado de salud buena o muy buena, asociada a la actividad física (23-24). Esto es acorde con los resultados de nuestro estudio, que encontró mayor prevalencia de inactividad física en los estados de salud regular y mala.

La principal conclusión del presente estudio es que la actividad física en las mujeres de Barranquilla se relaciona con el rango de edad, el estado civil, el nivel educativo y la localidad donde residen. Asimismo, las etapas de cambio y la autopercepción de salud están relacionadas con el hecho de ser activas e inactivas físicamente..

\section{Referencias}

1. Mantilla TS, Gómez CA, Hidalgo MM. Actividad física, tabaquismo y consumo de alcohol, en un grupo de estudiantes universitarios. Rev. salud pública 2011; 13(5): 748-758.

2. Organización Mundial de la Salud. Estrategia mundial sobre régimen alimentario, actividad física y salud. Inactividad física: un problema de salud pública mundial. OMS; 2013 consultado en Mayo de 2013; Disponible en: http://www.who.int/dietphysicalactivity/factsheet _inactivity/es/index.html\#

3. Sobejano TI, Moreno IC, Viñes RJ, Grijalba UA, Amézqueta GC, Serrano MM. Estudio poblacional de actividad física en tiempo libre. Gac Sanit. 2009; 23(2): 127-132.

4. Castro CJ, Patiño VF, Cardona RB, Ochoa PV. Aspectos Asociados a la Actividad Física en el Tiempo Libre en la Población Adulta de un Municipio Antioqueño. Rev. Salud pública. 2008; 10 (5): 679-690.

5. Bauman AE, Reis RS, Sallis JF, Wells JC, Loos RF, Martin BW. Correlates of physical activity: why are some people physically active and others not? Lancet. 2012; 380: 258-271.

6. Teixeira DC, Hernandes NA, Probst VS, Ramos EM, Brunetto AF, Pitta F. Profile of physical activity in daily life in physically independent elderly men and women. Rev. bras. educ. fís. esporte. 2012; 26 (4): 645-655.

7. Blazquez MA, Feu MS. Motivos de inscripción, permanencia y satisfacción en un programa de actividad física de mantenimiento para mujeres mayores. CPD. 2012; 12 (1): 79-92.

8. Vidarte JA, Vélez C, Parra JH. Niveles de sedentarismo en población de 18 a 60 años. Manizales, Colombia, Rev. salud pública. 14 (3): 417-428, 2012. 
9. Velez C, Vidarte JA, Ríos DM, Muñoz AP. Prevalencia de Actividad Física y factores asociados en la población de 18-60 años Pereira2010. Rev. Méd. Risaralda. 2011; 17 (2): 85-90.

10. Devís, J. Actividad física, deporte y salud $\left(2^{\mathrm{a}}\right.$ edición). Barcelona: INDE; 2007.

11. Vidarte JA, Vélez C, Montealegre LM. Modelo predictivo de los niveles de sedentarismo en población entre 18 y 60 años de la ciudad de Neiva. Rev. Entornos. 2012; 25: 196-211.

12. Venegas OU, Llerenas TC, Aguayo GA, Navarro SJ, Corbalá SJ, Campos A, et al. Actividad e inactividad física en las mujeres que trabajan en los servicios de salud, Ginecol Obstet Mex. 2006; 74 (9): 471-475.

13. Castro CJ, Patiño VF, Cardona RB, Ochoa PV. Aspectos asociados a la actividad física en el tiempo libre en una población adulta de un municipio antioqueño. Rev salud pública. 2008; 10(5):679-690.

14. Echeverri P, Zapata C, Giraldo J. Impacto de un modelo de movilización social sobre la promoción de la actividad física en afiliados al sistema se seguridad social en salud. Rev. salud pública. 2008; 10(3): 361-373.

15. Muévase contra el sedentarismo. Actividad Física y Desarrollo Humano. Facultad de Rehabilitación y Desarrollo Humano, Universidad del Rosario (Fascículo 7, Tomo II). Disponible en: www.urosario.edu.co/investigacion).

16. Grimaldo MP. Estilos de vidas saludables en un grupo de estudiantes de una universidad particular de la ciudad de Lima. Liberabit. 2005; 11: 75-82.

17. Robledo R. Características socioculturales de la actividad física en tres regiones de Colombia. Rev. Salud pública. 2006; 8 (Sup. 2):13-27.

18. Elizondo AJ, Guillen F, Aguinaga I. Prevalencia de la actividad Física y su relación con variables sociodemográficas y estilo de vida en la población de 18 a 65 años de Pamplona. Rev. Esp Salud Pública. 2005; 79 (5): 559-567.

19. Castillo G, Pachajoa H, Zurita E, Pradilla A. Identificación de factores de riesgo para enfermedades crónicas no transmisibles en estudiantes de medicina de la Universidad del Valle. CIMEL. 2005; 10 (2): 37-45.

20. Cordente CA, García P, Sillero M, Stirling J. Predicción del nivel de actividad física en adolescentes a partir de diversos factores biopsicosociales de influencia. $9^{\circ}$ Congreso
Gallego de Estadística e Investigación de Operaciones. Ourense; 2009.

21. Carlson JA, Sallis JF, Norman GJ, McKenzie TL, Kerr J, Arredondo EM, et al. Elementary school practices and children's objectively measured physical activity during school. Prev Med. 2013; 57(5): 591-595.

22. Olivares S, Lera L, Bustos N. Etapas del cambio, beneficios y barreras en actividad física $\mathrm{y}$ consumo de frutas y verduras en estudiantes universitarios en Santiago de Chile. Rev. chil. nutr. 2008; 35 (1): 25-35.

23. Ministerio de la Protección Social, Colciencias, Agudelo CA, Prieto A. Modelos de Movilización social con énfasis en actividad física y estilos de vida saludables para reducir el sedentarismo en las regiones de Bogotá D.C., Antioquia y Quindío. Bogotá D.C.: Universidad Nacional de Colombia; 2006.

24. Gómez LF, Duperly J, Lucumí DI, Gámez R, Venegas AS. Nivel de actividad física global en la población adulta de Bogotá (Colombia). Prevalencia y factores asociados. Gac.Sanit. 2005; 19 (3):206-213. 